Article

\title{
Surface Modeling from 2D Contours with an Application to Craniofacial Fracture Construction
}

\author{
Abdul Majeed ${ }^{1} \mathbb{D}$, Muhammad Abbas ${ }^{2,3} *^{*} \mathbb{D}$, Kenjiro T. Miura ${ }^{4} \mathbb{D}$, Mohsin Kamran ${ }^{1}$ \\ and Tahir Nazir 5
}

1 Department of Mathematics, Division of Science and Technology, University of Education, Lahore 54770, Pakistan; abdulmajeed@ue.edu.pk (A.M.); mohsin.kamran@ue.edu.pk (M.K.)

2 Informetrics Research Group, Ton Duc Thang University, Ho Chi Minh City 70000, Vietnam

3 Faculty of Mathematics and Statistics, Ton Duc Thang University, Ho Chi Minh City 70000, Vietnam

4 Department of Mechanical Engineering, Shizuoka University, Hamamatsu, Shizuoka 432-8011, Japan; miura.kenjiro@shizuoka.ac.jp

5 Department of Mathematics, University of Sargodha, Sargodha 40100, Pakistan; tahir.nazir@uos.edu.pk

* Correspondence: muhammadabbas@tdtu.edu.vn

Received: 1 July 2020; Accepted: 25 July 2020; Published: 30 July 2020

\begin{abstract}
Treating trauma to the cranio-maxillofacial region is a great challenge and requires expert clinical skills and sophisticated radiological imaging. The aim of reconstruction of the facial fractures is to rehabilitate the patient both functionally and aesthetically. Bio-modeling is an important tool for constructing surfaces using $2 \mathrm{D}$ cross sections. The aim of this manuscript was to show 3D construction using 2D CT scan contours. The fractured part of the cranial vault were constructed using a Ball curve with two shape parameters, later the 2D contours were flipped into 3D with an equidistant $z$ component. The surface created was represented by a bi-cubic rational Ball surface with $C^{2}$ continuity. At the end of this article, we present two real cases, in which we had constructed the frontal and parietal bone fractures using a bi-cubic rational Ball surface. The proposed method was validated by constructing the non-fractured part.
\end{abstract}

Keywords: CT scan data; rational Ball curves; bi-cubic Ball surface; 2D fractured part curves; 3D craniofacial reconstruction

\section{Introduction}

The etiology of craniofacial fractures stretch from road traffic accidents, fist fights, falls and sports injury. Craniofacial fractures are a common site when compared with fractures of the rest of the body.Craniofacial fractures are more sensitive and complicated than fractures on other parts of the body. The complexity of the craniofacial region and the random complex patterns of its fractures are always a challenge to diagnose and treat. To diagnose a craniofacial fracture, various tools are available, such as MRIs, X-rays and CT scans. To understand the complexity of the maxillo-craniofacial region, Figure 1 explains the various bones. The most common bones included in the region are maxilla, mandible, frontal bone, orbital cavity (made of nine bones), nasal cavity, and parietal bone. In this manuscript, the cases included had frontal and parietal bone fractures.

Emerging virtual reconstruction technologies opened new avenues for mathematicians, physicists and software engineers to reconstruct the fracture defects. The already established approaches for implant design are based on the Computer Aided Design (CAD)/Computer Aided Manufacturing (CAM) process chain [1,2]. Heavy tools and technical staff are required for this process, which causes high cost and low efficiency. Sauret et al. [3] proposed the mirroring method. The mirroring method is useful for the fracture on one side of the skull only. The authors in [4] used 
the adaptive deformation method for fracture reconstruction. In this method, firstly, the author has constructed a reference model according to patients skull, and, later, performed a $3 D$ matching for the correlation.

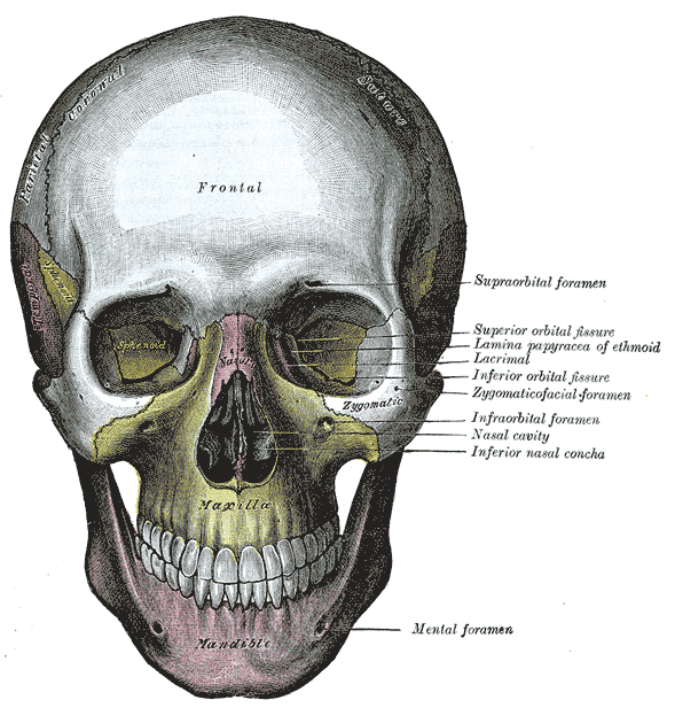

Figure 1. Craniofacial bones.

The method, based on a reference skull, is used by [5] for fracture reconstruction. By this method, a user can not get custom made implants. Carr et al. [6,7] used the Radial Basis Functions (RBF) for the reconstruction and representation of $3 D$ objects. Multiple bone fractures in $2 D$ were constructed by [8] using Non-Uniform Rational B-Spline (NURBS) curves. Occipital bone fractures were constructed by [9] using B-spline curves. Rational Ball curves were used in [10] for image reconstruction and have constructed different images like a Bear, vase and the Arabic word "Dal". A virtual craniofacial model was constructed by [11] using an algorithm based on the Iterative Closest Point (ICP). The author constructed a mandible bone fracture using this method. King et al. [12] explained the statistical analysis of a patient with a mandible bone fracture based on the age and gender of patients, and the causes of fractures. Pascoletti et al. [13] constructed a 3D mandible model by using a limited number of control points through principal component analysis. Computerized craniofacial reconstruction was done by [14] using a CT-derived implicit surface representation. Klein et al. [15] used contours to reconstruct the fractures. A dense surface point distribution model was used by [16] for anatomical shape reconstruction. The authors in $[17,18]$ used a reverse engineering method for the construction of different parts of the human body. Lian et al. [19] used a computer modeling approach for the construction of artificial human bones.

In our study, we used a bi-cubic rational Ball surface to reconstruct the 3D fractured craniofacial site. The given CT scan Digital Imaging and Communications in Medicine (DICOM) data are in $2 D$ form, so first we construct the fracture part curves of skull in its 2D form, using rational cubic curves based on the Ball basis. The free parameters were optimized using the Genetic Algorithm (GA). Then, we converted the given $2 D$ data into $3 D$ form, taking the equidistant $z$ component and then constructed the $3 D$ craniofacial fracture.

Using the proposed method, there is no need to use a reference model, mirroring, statistical analysis or technical staff. The construction is just based on the CT scan data in 2D format. The proposed method provides a custom made implant for every unique patient. Table 1 represents the comparison between the existing method and proposed method. To validate the proposed method, real cases with frontal and parietal bone fractures were used in this paper. 
Table 1. Comparison of existing methods and proposed method.

\begin{tabular}{|c|c|c|}
\hline Sr.No & Existing Methods & Proposed Method \\
\hline 1 & $\begin{array}{l}\text { The method based on CAD/CAM is } \\
\text { used by }[1,2] \text { for fracture reconstruction. } \\
\text { Technical staff/tools are required for this } \\
\text { method, and this method suffers from a } \\
\text { high cost and low efficiency }\end{array}$ & $\begin{array}{l}\text { In the proposed method, there is no need for } \\
\text { staff/tools, only a patient's CT scan DICOM } \\
\text { data are required for construction. }\end{array}$ \\
\hline 2 & $\begin{array}{l}\text { The authors in [3] constructed a fractured part } \\
\text { using the mirroring method. This method } \\
\text { works well for unilateral fractures and will } \\
\text { not work for bilateral fractures }\end{array}$ & $\begin{array}{l}\text { The proposed method works independently } \\
\text { of mirroring and will work well for both } \\
\text { unilateral and bilateral fractures. }\end{array}$ \\
\hline 3 & $\begin{array}{l}\text { Wu et al. [4] and Shui et al. [5] used the } \\
\text { adaptive deformation method for construction. } \\
\text { This method is based on a reference skull }\end{array}$ & $\begin{array}{l}\text { There is no need for a reference skull using the } \\
\text { proposed method, only } 2 D \text { CT scan data are } \\
\text { required. }\end{array}$ \\
\hline 4 & $\begin{array}{l}\text { Shui et al. [5] used a thin plate spline; this } \\
\text { method also depends on a reference skull }\end{array}$ & $\begin{array}{l}\text { The proposed method is independent of } \\
\text { reference skull construction and directly uses } \\
\text { patient data. }\end{array}$ \\
\hline 5 & $\begin{array}{l}\text { Carr et al. [6] employed radial basis function. } \\
\text { A large number of data points are required } \\
\text { for this method and they use the average } \\
\text { thickness of skull bone }\end{array}$ & $\begin{array}{l}\text { The thickness of bone varies slice to slice and } \\
\text { can be controlled using the free parameters } \\
\text { of proposed method with no need for } \\
\text { average thickness. }\end{array}$ \\
\hline 6 & $\begin{array}{l}\text { A mandible bone fracture was constructed } \\
\text { by King et al. [12] using ICP, taking the } \\
\text { non-fractured part as a reference }\end{array}$ & $\begin{array}{l}\text { The proposed method is independent of a } \\
\text { reference skull. The constructed fractured } \\
\text { part can be controlled and adjusted by shape } \\
\text { parameters in proposed method. It is a custom } \\
\text { made implant, time-saving and efficient. }\end{array}$ \\
\hline 7 & $\begin{array}{l}\text { Majeed et al. }[8,9] \text { used NURBS and B-spline } \\
\text { curves for the construction of multiple and } \\
\text { occipital bones fractures. The constructed } \\
\text { parts in both papers are in } 2 D \text { form. }\end{array}$ & $\begin{array}{l}\text { In this paper, we constructed the frontal and } \\
\text { parietal bone fractures in } 3 D \text { form using the } \\
\text { bi-cubic Ball surface. }\end{array}$ \\
\hline
\end{tabular}

\section{The Cubic Ball Basis Functions, Curves and Surfaces}

In this section, we construct the cubic Ball curves and surfaces using Ball basis functions [20]. These functions satisfy the basic properties like symmetry, invariant under affine transformation, coordinate system independence, convex hull property, end point interpolation and variation diminishing property. In this paper, we used the cubic Ball basis functions for fracture reconstruction. Figure 2 explains the graphical behavior of these functions. The cubic ball basis functions are defined as:

$$
\left\{\begin{array}{l}
\Im_{0}(\psi)=(1-\psi)^{2} \\
\Im_{1}(\psi)=2 \psi(1-\psi)^{2}, \\
\Im_{2}(\psi)=2 \psi^{2}(1-\psi), \\
\Im_{3}(\psi)=\psi^{2}
\end{array}\right.
$$




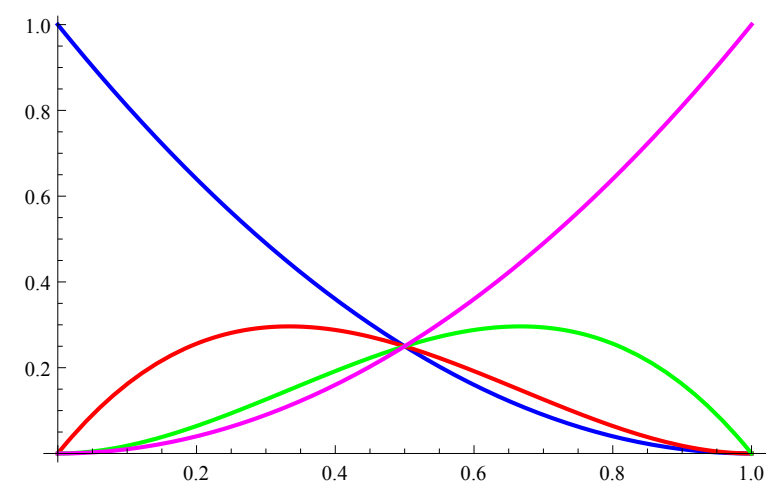

Figure 2. Ball basis functions.

\subsection{Properties of Ball Basis Functions and Curve}

- Linearly Independent: The cubic Ball bases are linearly independent. We can not get constants $a_{i} \neq 0$, for which.

$$
\sum_{i=0}^{3} a_{i} \Im_{i}(\psi)=0
$$

- Non-negative: Cubic Ball functions are always positive for $\psi \in[0,1]$.

- Symmetric: The cubic Ball functions are symmetric as

$$
\Im_{i}(\psi)=\Im_{3-i}(1-\psi) .
$$

- Monotonicity: $\Im_{3}(\psi)$ is monotonically increasing and $\Im_{0}(\psi)$ is monotonically decreasing for $\psi \in[0,1]$.

- Partition of Unity: Sum of cubic Ball basis functions is 1 .

$$
\sum_{i=0}^{3} \Im_{i}(\psi)=1
$$

Let $\aleph_{i}$ be the set of control points and $\Im_{i}(\psi), i=0, \ldots, 3$ are cubic Ball functions defined in (1). The cubic Ball curve $\Im(\psi)=\sum_{i=0}^{3} \aleph_{i} \Im_{i}(\psi)$ obey the following properties [21,22]

- Coordinate system independence: The Ball curve is independent of coordinate systems. By changing the coordinates of control points curve remains same.

- Convex Hull Property: The Ball curve obeys the convex hull property means curve will always lies within the convex hull of its control polygon.

- Variation Diminishing Property (VDP): Variation Dimension Property is obeyed by Ball curves as shown in Figure 3.

- Endpoint Interpolation: The cubic Ball curve interpolates the first and last control point. $\Im(0)=\aleph_{0}, \Im(1)=\aleph_{3}$. 


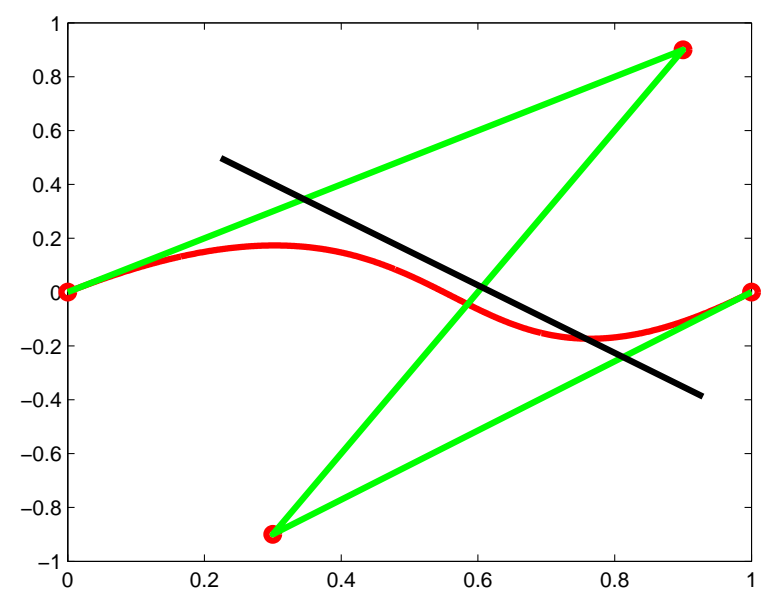

Figure 3. Variation diminishing property with $b=1 p=3 k=1$.

\subsection{The Rational Cubic Ball Curve}

A rational cubic Ball curve used for the fracture reconstruction is defined as:

$$
\Im_{i}(\psi)=\frac{\aleph_{i}(\psi)}{\mathrm{C}_{i}(\psi)}, i=1, \ldots n-1 \text { with } 0 \leq \psi \leq 1,
$$

where,

$$
\left\{\begin{array}{l}
\aleph_{i}(\psi)=\hbar_{i}(1-\psi)^{2}+V_{i}(1-\psi)^{2} \psi+W_{i}(1-\psi) \psi^{2}+\hbar_{i+1} \psi^{2} \\
\aleph_{i}(\psi)=(1-\psi)^{2}+a_{i}(1-\psi)^{2} \psi+b_{i}(1-\psi) \psi^{2}+\psi^{2}
\end{array}\right.
$$

and $0=\psi_{0}<\psi_{1}<\psi_{2}<\psi_{3} \ldots .<\psi_{n}=1, V_{i}=a_{i} \hbar_{i}+h_{i} d_{i}, W_{i}=b_{i} \hbar_{i+1}-h_{i} d_{i+1}$.

Equation (2) satisfies the following conditions

$$
\begin{cases}\Im_{i}\left(\psi_{0}\right)=\hbar_{i}, & \Im_{i}\left(\psi_{n}\right)=\hbar_{i+1}, \\ \Im_{i}^{\prime}\left(\psi_{0}\right)=d_{i}, & \Im_{i}^{\prime}\left(\psi_{n}\right)=d_{i+1} .\end{cases}
$$

where $a_{i}, b_{i}$ are free parameters, $\hbar_{i}, \hbar_{i+1}$ are the endpoints of each segment, and $d_{i}, d_{i+1}$ are unit tangent vectors at $\hbar_{i}$ and $\hbar_{i+1}$, respectively.

The given DICOM data are in $2 D$ form. To construct the surface patch one have to convert the data in $3 D$ form, for this we took the non-decreasing $z$ component of each $2 D$ contours as a height like $z_{0}<z_{1}<\ldots<z_{\max }$. The $i$ th contour is defined by the sequence of distinct data points, which are counterclockwise ordered in the contour at the height $z_{i}$.

\subsection{Bi-Cubic Rational Ball Surface}

Frontal and parietal bone fractures are constructed using bi-cubic rational Ball surface, which is defined as:

$$
\Im_{i j}(x, y)=\frac{X B B^{*} B^{T} Y^{T}}{\rho(x) \zeta(y)}
$$

where,

$$
\begin{aligned}
& X=\left[\begin{array}{llll}
x^{3} & x^{2} & x & 1
\end{array}\right] \\
& Y=\left[\begin{array}{llll}
y^{3} & y^{2} & y & 1
\end{array}\right]
\end{aligned}
$$

The Matrix $B$ is the Ball matrix

$$
\rho(x)=(1-x)^{2}+\alpha_{i} u(1-x)^{2}+\beta_{i} x^{2}(1-x)+x^{2},
$$




$$
\zeta(y)=(1-y)^{2}+\alpha_{j} y(1-y)^{2}+\beta_{j} y^{2}(1-y)+y^{2}
$$

and

$$
B^{*}=\left[\begin{array}{cccc}
\hbar_{i, j}^{*} & \hbar_{i, j+1}^{*} & \hbar_{i, j}^{* y} & \hbar_{i, j+1}^{* y} \\
\hbar_{i+1, j}^{*} & \hbar_{i+1, j+1}^{*} & \hbar_{i+1, j}^{* y} & \hbar_{i+1, j+1}^{*} \\
\hbar_{i, j}^{* x} & \hbar_{i, j+1}^{* x} & \hbar_{i, j}^{* x y} & \hbar_{i, j+1}^{* x y} \\
\hbar_{i+1, j}^{* x} & \hbar_{i+1, j+1}^{* x} & \hbar_{i+1, j}^{* x y} & \hbar_{i+1, j+1}^{* x y}
\end{array}\right]
$$

where $\hbar_{i j}^{*}$ are the control points of the curves along $x$ and $y$ direction. $\hbar_{i j}^{x}, \hbar_{i j}^{y}$ are the first derivatives along $x$ and $y$ defined as follows

$$
\left\{\begin{array}{l}
\hbar_{00}^{* x}=2\left(\hbar_{10}^{*}-\hbar_{00}^{*}\right)-\left(\hbar_{20}^{*}-\hbar_{00}^{*}\right) / 2, \\
\hbar_{00}^{* y}=2\left(\hbar_{01}^{*}-\hbar_{00}^{*}\right)-\left(\hbar_{02}^{*}-\hbar_{00}^{*}\right) / 2, \\
\hbar_{n m}^{* x}=2\left(\hbar_{n m}^{*}-\hbar_{n-1 m}^{*}\right)-\left(\hbar_{n m}^{*}-\hbar_{n-2 m}^{*}\right) / 2, \\
\hbar_{n m}^{* y}=2\left(\hbar_{n m}^{*}-\hbar_{n m-1}^{*}\right)-\left(\hbar_{n m}^{*}-\hbar_{n m-2}^{*}\right) / 2, \\
\left.\hbar_{i j}^{* x}=a_{i j}^{*} \hbar_{i j}^{*}-\hbar_{i-1 j}^{*}\right)-\left(1-a_{i j}^{*}\right)\left(\hbar_{i+1 j}^{*}-\hbar_{i j}^{*}\right), \quad i=1, \ldots n-1, j=1, \ldots m-1 \\
\hbar_{i j}^{* y}=a_{i j}^{* *}\left(\hbar_{i j}^{*}-\hbar_{i j-1}^{*}\right)-\left(1-a_{i j}^{* *}\right)\left(\hbar_{i j+1}^{*}-\hbar_{i j}^{*}\right),
\end{array}\right.
$$

where,

$$
\begin{gathered}
a_{i j}^{*}=\frac{\left|\hbar_{i+1 j}^{*}-\hbar_{i j}^{*}\right|}{\left|\hbar_{i+1 j}^{*}-\hbar_{i j}^{*}\right|+\left|\hbar_{i j}^{*}-\hbar_{i-1 j}^{*}\right|}, \quad i=1, \ldots n, j=1, \ldots m \\
a_{i j}^{* *}=\frac{\left|\hbar_{i j+1}^{*}-\hbar_{i j}^{*}\right|}{\left|\hbar_{i j+1}^{*}-\hbar_{i j}^{*}\right|+\left|\hbar_{i j}^{*}-\hbar_{i j-1}^{*}\right|},
\end{gathered}
$$

where $\hbar_{i j}^{* x y}$ are the second derivatives defined [23] as:

$$
\hbar^{*}(x, y)=\hbar_{i j}^{*}(1-x)(1-y)+\hbar_{i j+1}^{*}(1-x) y+\hbar_{i+1 j}^{*} x(1-y)+\hbar_{i+1 j+1}^{*} x y .
$$

Partially differentiate Equation (5) with respect to $x$ and $y$, we get

$$
\hbar_{i j}^{* x y}=\left(\hbar_{i j}^{*}-\hbar_{i j+1}^{*}\right)+\left(\hbar_{i+1 j+1}^{*}-\hbar_{i+1 j}^{*}\right) .
$$

For more detail, readers can see [23]. The method proposed in this section has shape parameters, which help in adjusting and changing the surface.

\section{Craniofacial Fractures Reconstruction}

This section discusses the validity of proposed method, $3 D$ fracture reconstruction and the proposed algorithm.

\subsection{Validity of Proposed Method}

The proposed method was validated by reconstructing the non-fractured part of real data as shown in Figure 4. Figure 4a shows that around one fourth of the data are non-fractured. We have successfully constructed that part using the proposed bi-cubic rational Ball surfaces as shown in Figure $4 b$. 


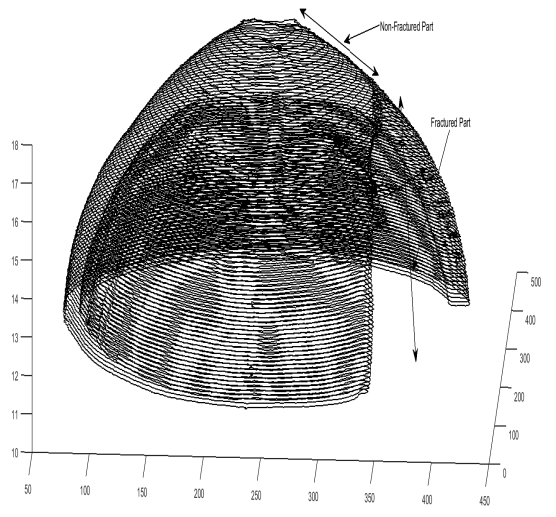

(a) CT scan data in 3D form

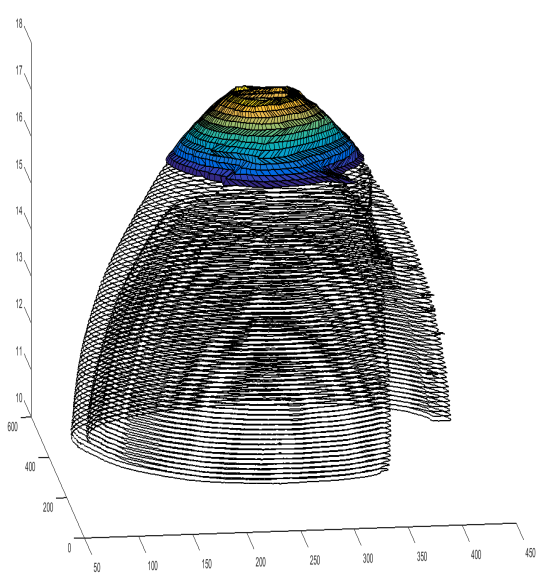

(b) Non-fractured part reconstruction

Figure 4. Validity of the proposed method.

\subsection{Reconstruction Accuracy}

Normalized mean squares error is used to find the error of constructed surface as in Figure 4. The normalized mean squares error is computed by

$$
E^{2}=\frac{\sum\left|\Im_{i j}(x, y)-D_{i}\right|^{2}}{\sum\left|D_{i}\right|^{2}}
$$

The errors for different patches of constructed surface lies between $10^{-3}$ to $10^{-1}$. The average error for complete surface is about $10^{-2}$.

\subsection{Case Study: 3D Craniofacial Fractures Construction}

In this section, the frontal and parietal bone fractures were constructed in $3 D$ form using proposed bi-cubic Ball surface. The given 2D CT scan data are in slices, as shown in Figure 5. A flow chart for craniofacial fracture reconstruction is shown in Figure 6. First, we constructed the boundary curves of a complete skull using the rational cubic Ball with $C^{1}$ continuity at each knot as shown in Figure 7 . Then, the rational cubic Ball curve with $C^{1}$ continuity was used to construct the boundary curves of fractured part of all CT scan slices, as shown in Figure 8. Figure 8a shows CT scan slice 171. For reconstruction, first we extracted the boundary of each CT scan slice using mathematical morphology as shown in Figure $8 \mathrm{~b}$. Then, rational cubic Ball curves were used to construct the fractured part boundary curves, as shown in Figure 8c. In a similar way, the boundary curves of different slices were constructed, as shown in Figures 9 and 10. To construct the fractured part in $3 D$ form, $2 D$ contours were switched to $3 D$ form by taking equidistant $z$ component, as shown in Figure 11. Figures 12 and 13 represent the constructed 3D craniofacial fracture using the proposed scheme.

The proposed method was applied to the patient with a parietal bone fracture, as shown in Figure 14. The rational cubic Ball curve was used to construct the boundary curves of the fractured part, as shown in Figure 15. The constructed boundary curves of the parietal bone fracture for different slices is shown in Figure 16. The effect of shape parameters are observed and shown in Figure 17. It is observed that shape parameters have a good effect on fracture reconstruction and by increasing the value of shape parameters the curves moves toward out side and curves move inner side by decreasing the values. Figure 18 represents the parietal bone fracture in $3 D$ form with and without fractured part curves. The constructed fracture part of parietal bone is represented by Figure 19. Two different craniofacial fractures have been constructed using the proposed method to show its applicability. The constructed part can be changed or adjust by using free parameters. 
The execution time for the construction of fractured part curves of one CT scan image is $0.03 \mathrm{~s}$ and for $3 D$ craniofacial fracture reconstruction is $2.59 \mathrm{~s}$. The system used for this work had a Processor $2.80 \mathrm{GHz}$; RAM $8 \mathrm{~GB}$; 64-bit operating system. Matlab was used for programming.

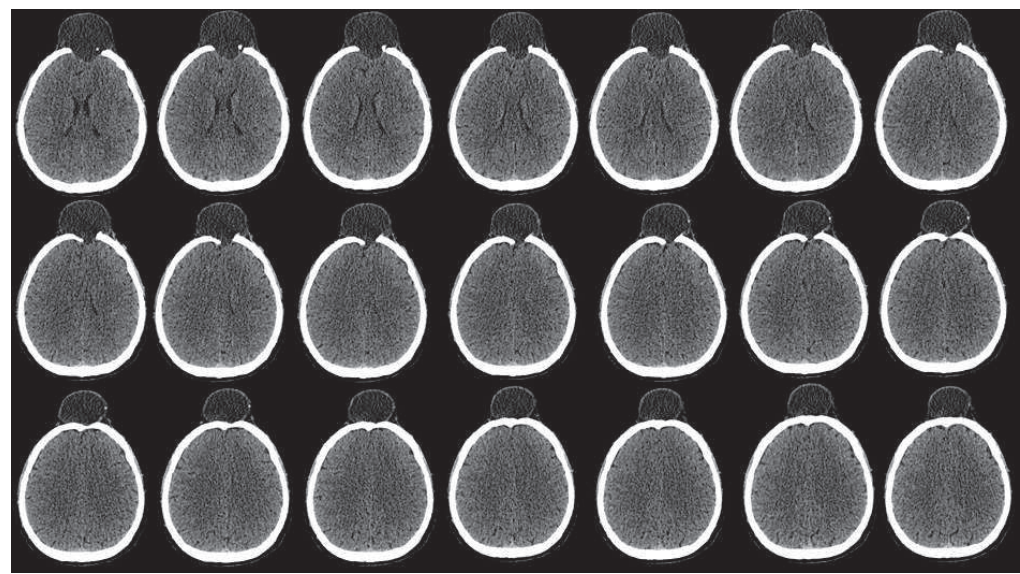

Figure 5. CT scan images of patient.

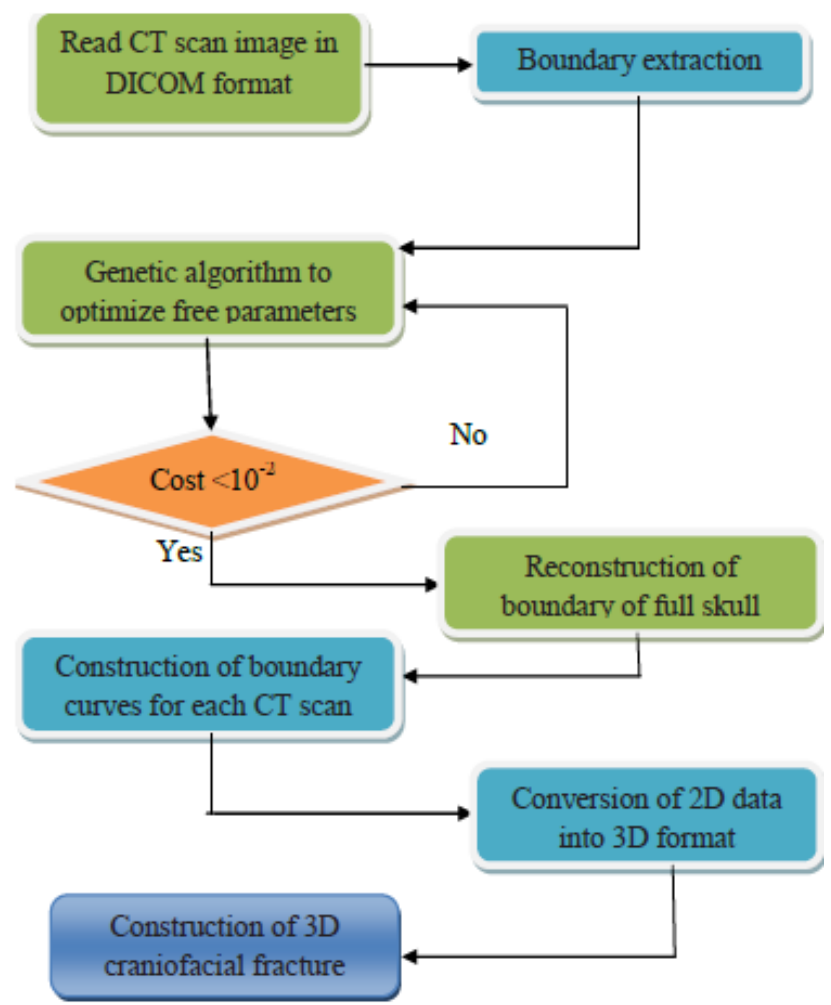

Figure 6. Flow chart of proposed algorithm. 


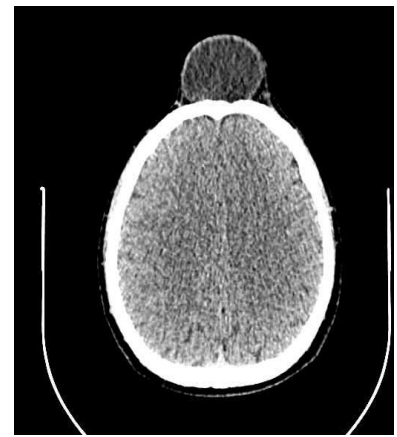

(a) Original image

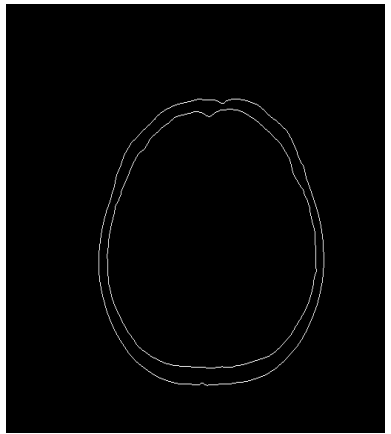

(b) Boundary

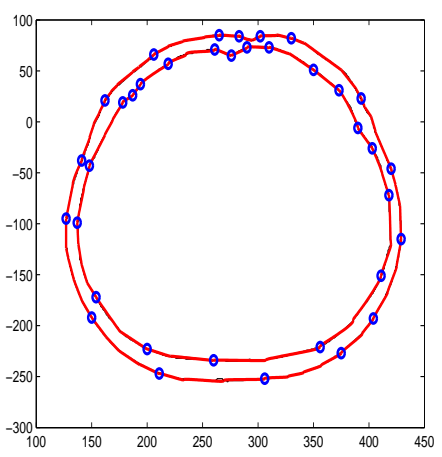

(c) Reconstructed image using rational Ball interpolant

Figure 7. Reconstructed image of CT scan data slice 176.

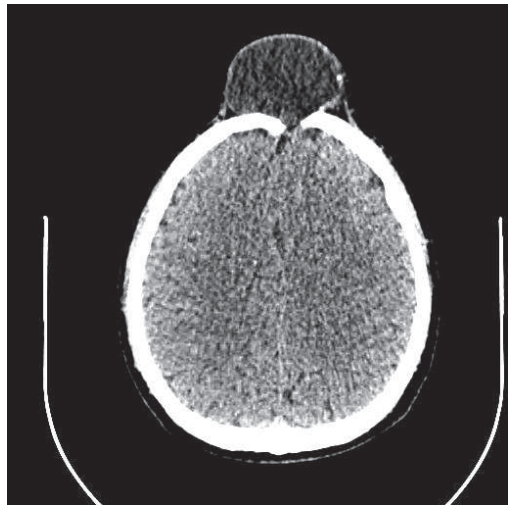

(a) Original image

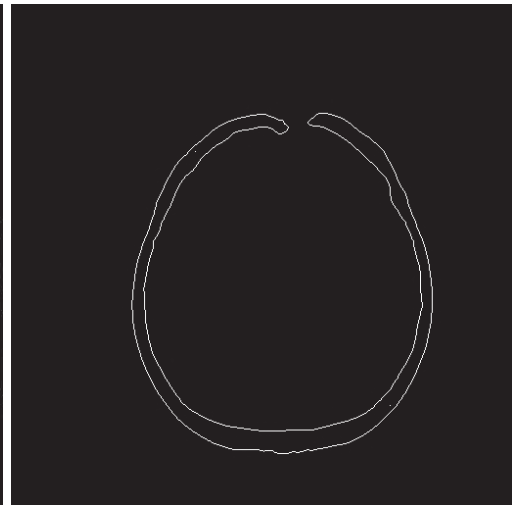

(b) Boundary

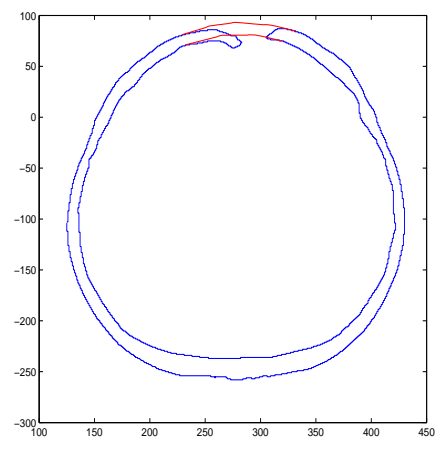

(c) Constructed fracture part curves of CT scan data slice 171

Figure 8. CT scan data slice 171.

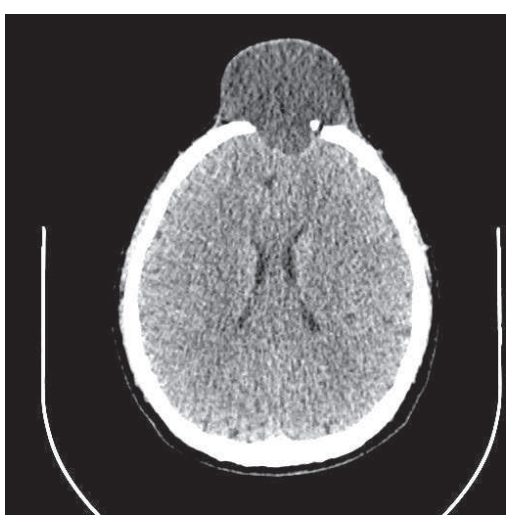

(a) Original image

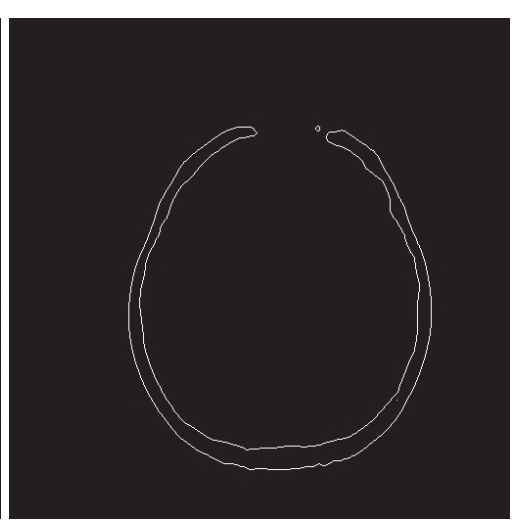

(b) Boundary

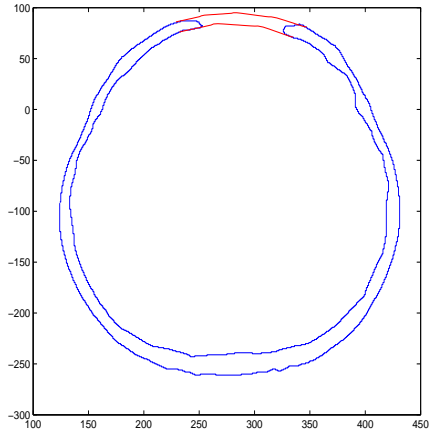

(c) Constructed fracture part curves of CT scan data slice 160

Figure 9. CT scan data slice 160. 


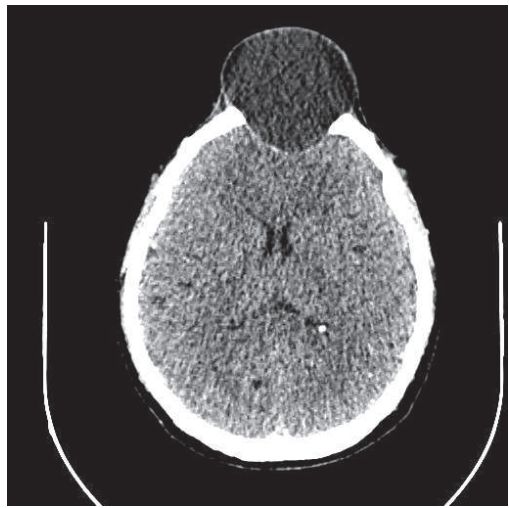

(a) Original image

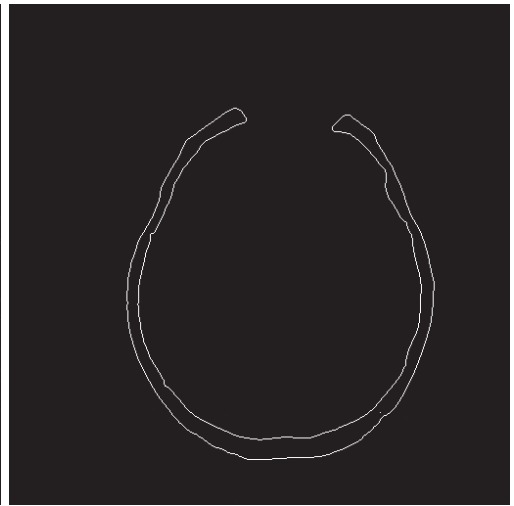

(b) Boundary

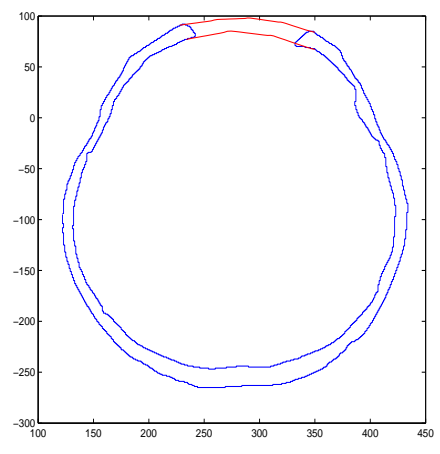

(c) Constructed fracture part curves of CT scan data slice 147

Figure 10. CT scan data slice 147.

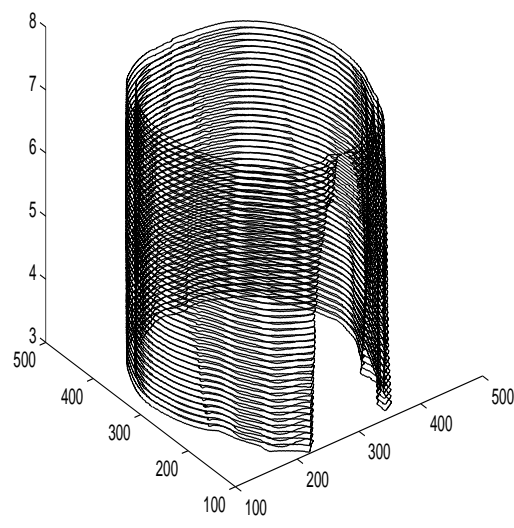

(a) CT scan data in 3D form

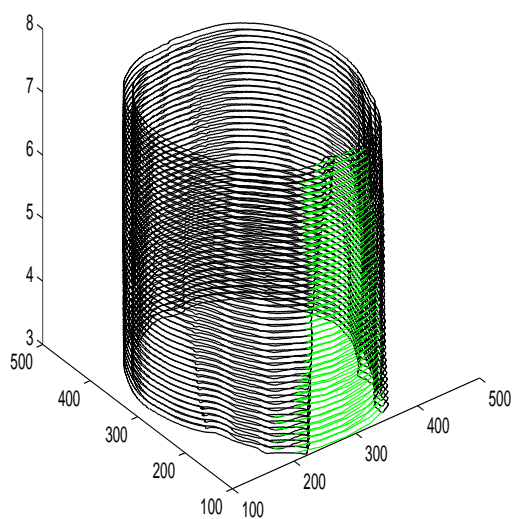

(c) Fractured part curves in 3D form

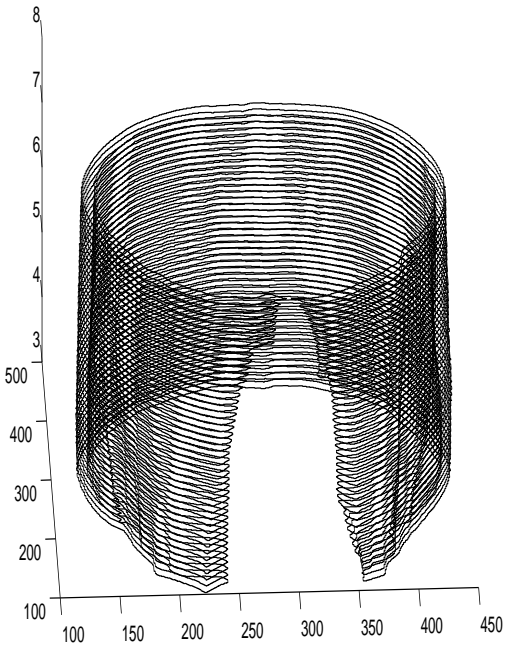

(b) CT scan data in 3D form

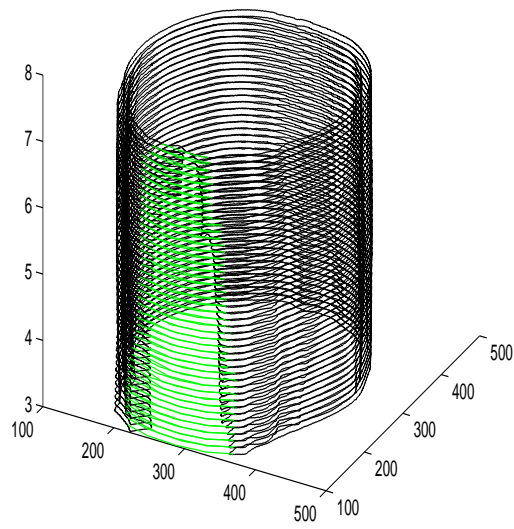

(d) Fractured part curves in 3D form

Figure 11. CT scan data in $3 \mathrm{D}$ format. 


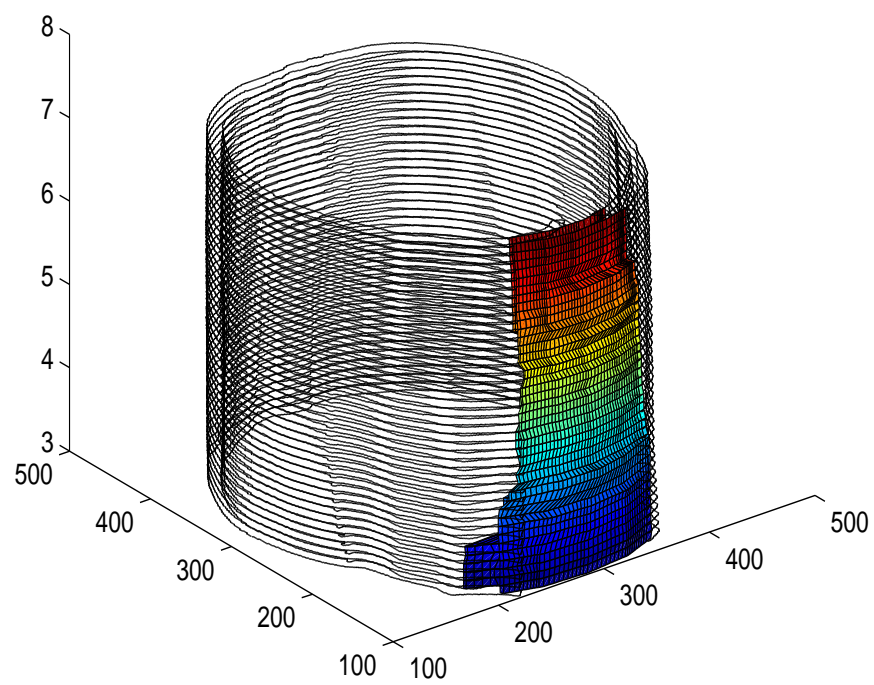

Figure 12. Reconstructed frontal bone in 3D.

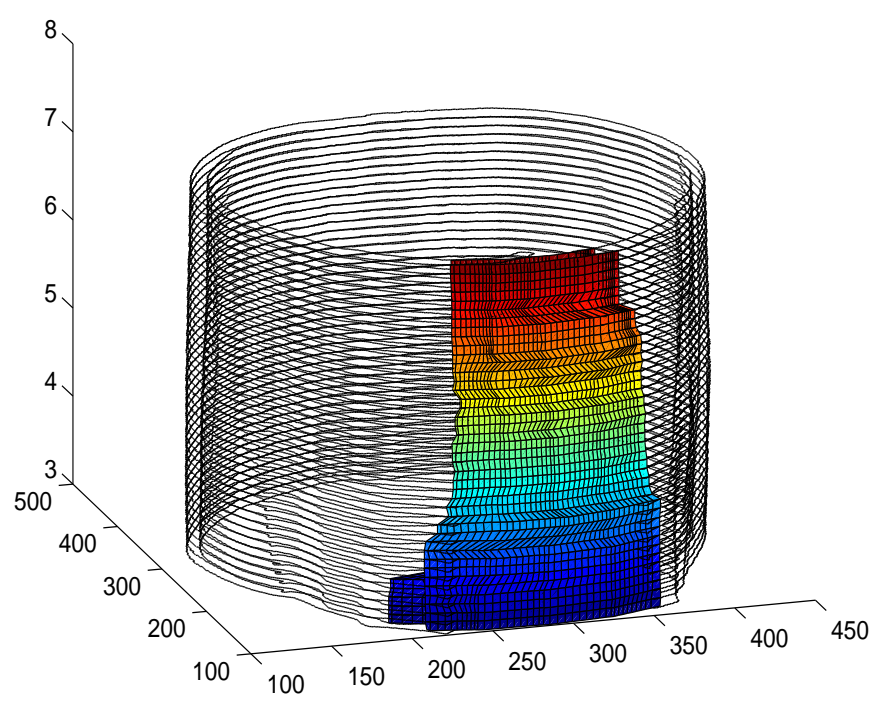

Figure 13. Reconstructed frontal bone in 3D.

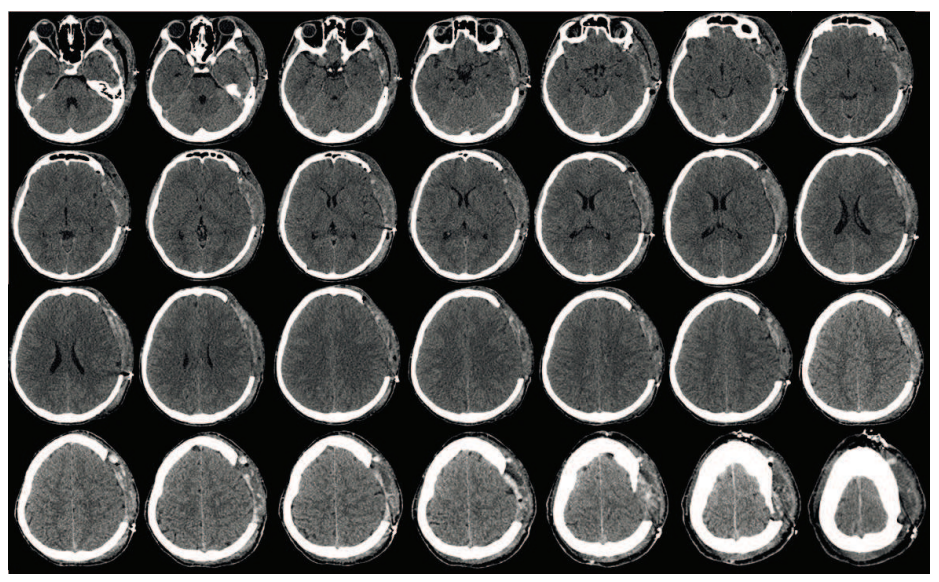

Figure 14. CT scan images of patient with parietal bone fracture. 


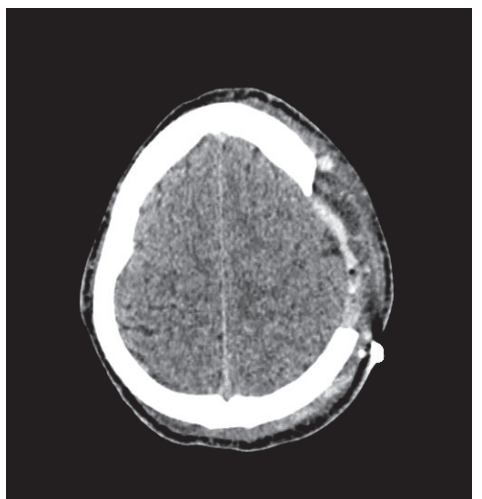

(a) Original image of parietal bone fracture

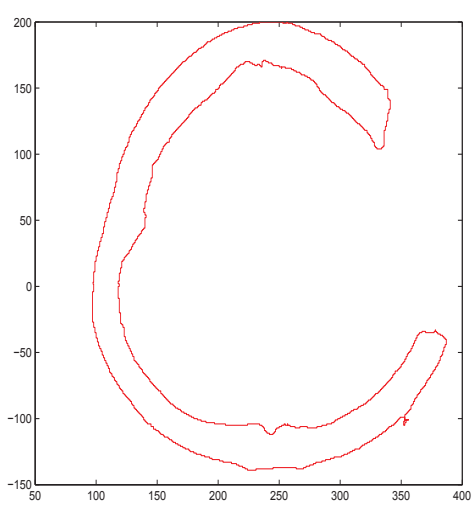

(b) Boundary of parietal bone fracture

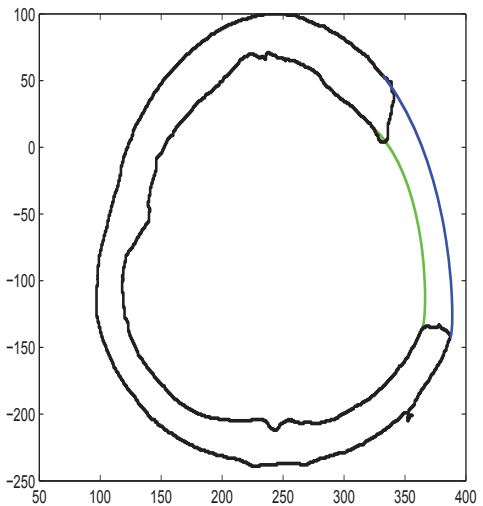

(c) Parietal bone fractured part curves construction

Figure 15. Construction of parietal bone fractured part curves using rational cubic Ball curves.
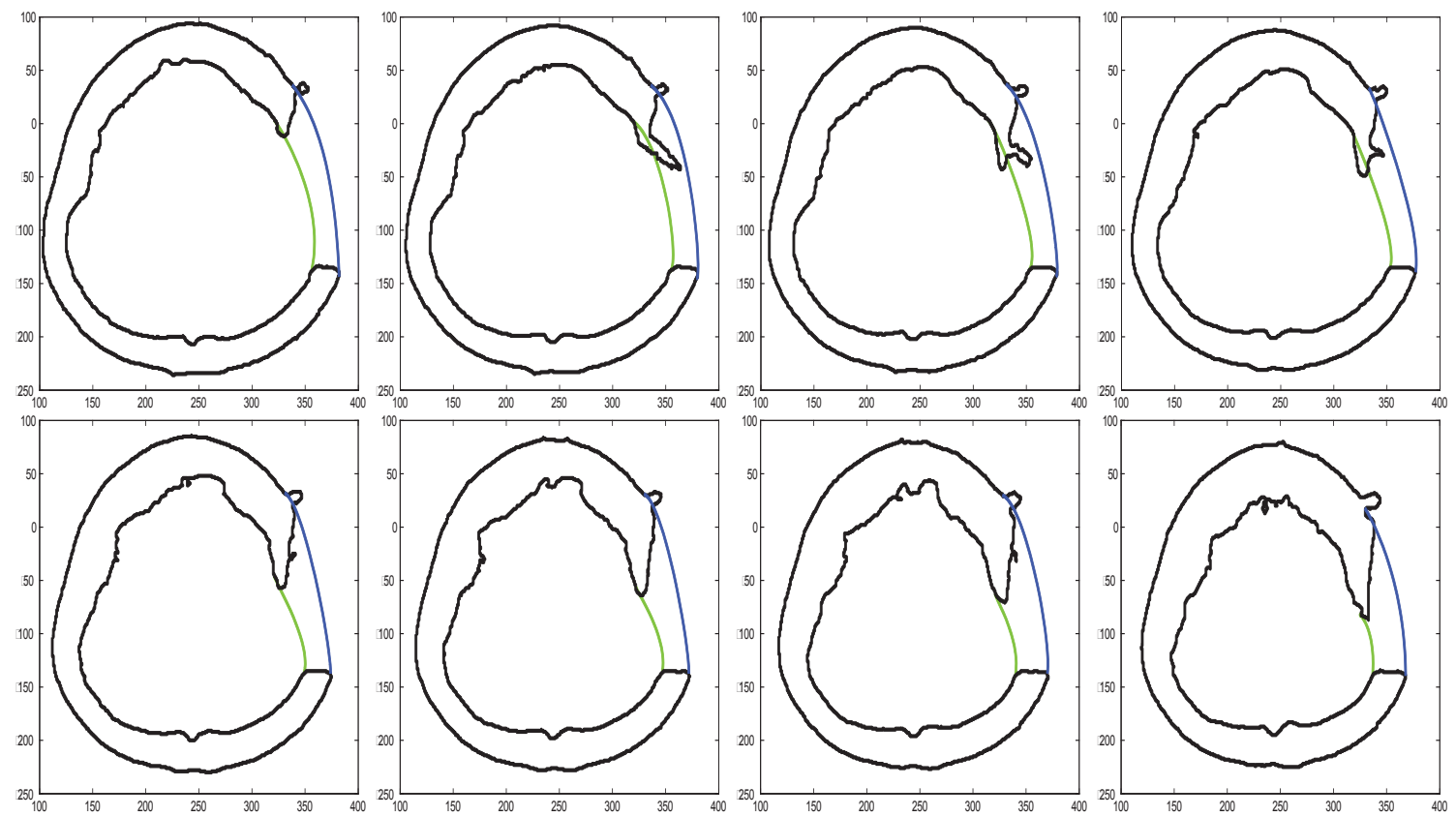

Figure 16. Construction of fractured parietal bone curves for different CT scan slices using a rational cubic Ball curves.

\subsection{Proposed Algorithm}

The algorithm for craniofacial fracture reconstruction is explained in this section. A flow chart for the proposed algorithm is shown in Figure 6.

1. Input: 2D CT scan DICOM data.

2. Output: Craniofacial fracture reconstruction in 3D form.

3. Read CT scan image as in Figure 5.

4. Boundary extraction as in Figure $7 \mathrm{~b}$.

5. Each segment is fitted using rational Ball interpolant. The unknown parameters $a_{i}, b_{i}$ in (2) are optimized using genetic algorithm (Figure 7c).

6. Step 5 is repeated until a desired solution is obtained.

7. Reconstruction of fractured part boundary curves for each CT scan slice.

8. Swapping 2D CT scan DICOM data to 3D form Figure 11. 
9. 3D craniofacial fracture reconstruction Figures 12 and 13 and Figure 19.

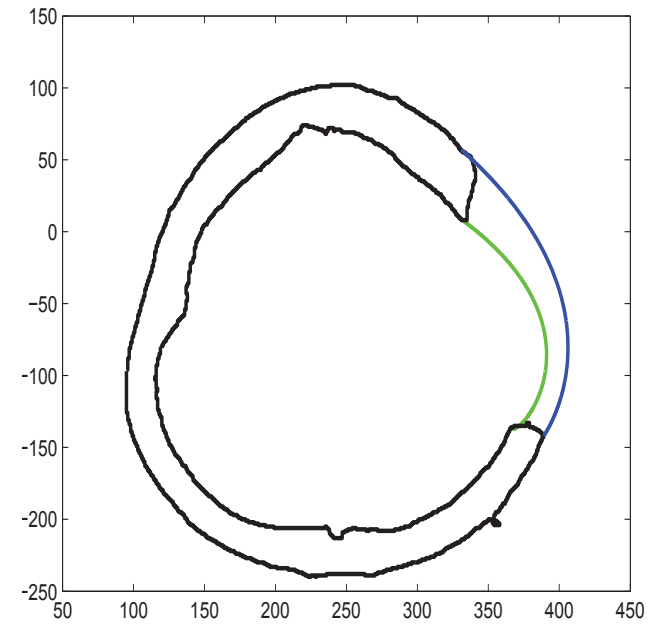

(a) inner $a=2.123, b=2.39$, outer $a=2.012$, $b=2.10$

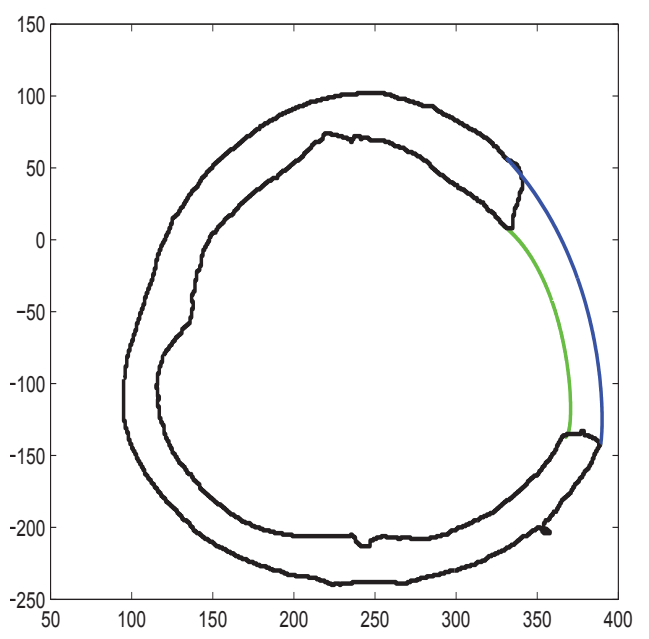

(c) inner $a=2.10, b=2.12$, outer $a=2.04, b=$ 2.029

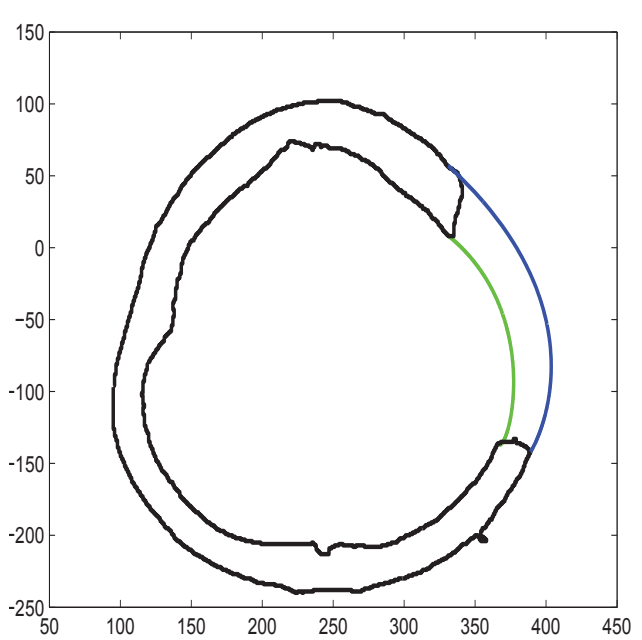

(b) inner $a=2.10, b=2.23$, outer $a=2.02, b=2.09$

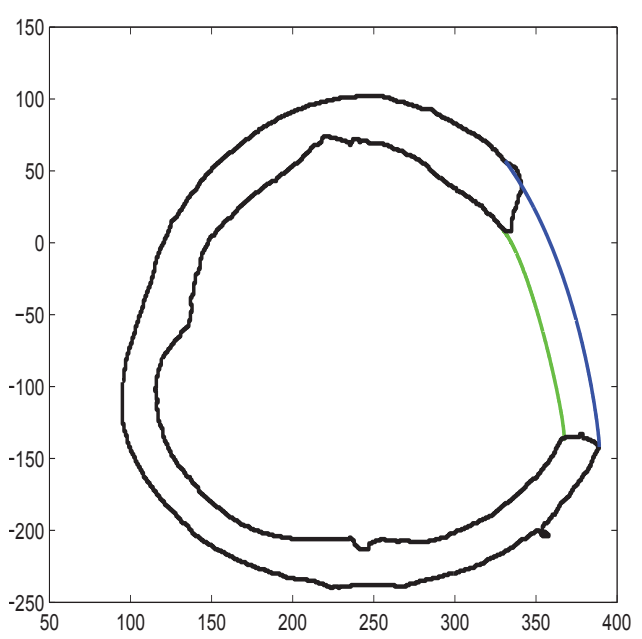

(d) inner $a=2.0199, b=2.010$, outer $a=2.001$, $b=2.0012$

Figure 17. Effect of shape parameters.

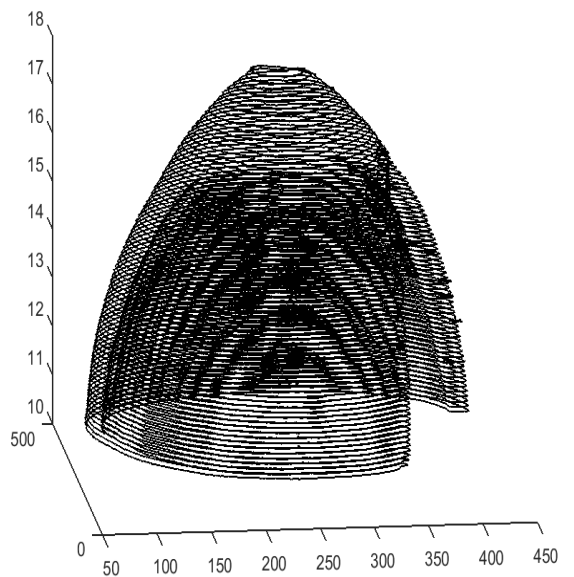

(a) Parietal bone fracture CT scan data in 3D form

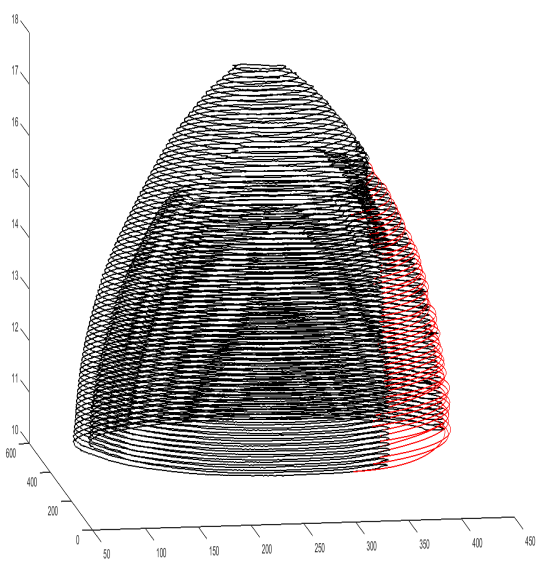

(b) Fractured part curves in 3D form

Figure 18. Parietal bone fracture data in 3D form. 


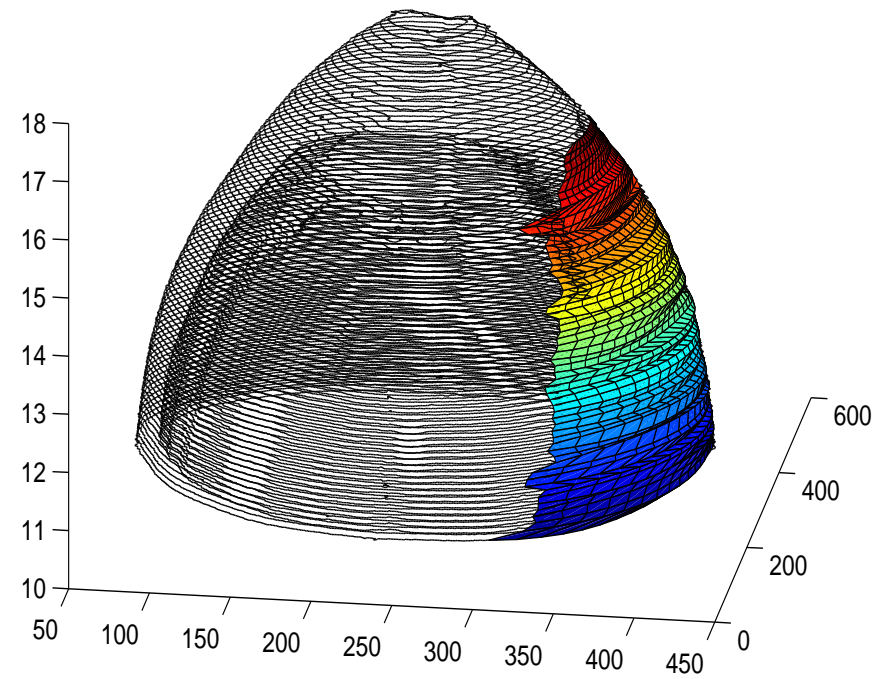

Figure 19. Reconstructed 3D craniofacial fracture of parietal bone fracture.

\section{Conclusions}

This manuscript proposed a bi-cubic Ball surface for the construction of 3D craniofacial fractures. One patient with a frontal bone fracture and another with a parietal bone fracture as case studies were illustrated to show its applicability. The given data was in $2 D$ form so first we constructed the fracture part curve of each contour in $2 D$ form using rational Ball curves. Then, we converted it into $3 D$ form taking equidistant $z$ component for fracture reconstruction. The genetic algorithm was used to optimize the free parameters. The proposed method saves time because, in this method, there is no need to use a reference skull, mirroring or any technical support. Using this method, any type of fracture can be constructed, and we applied this method on frontal and parietal bone fractures successfully. The constructed fracture is flexible due to the presence of shape parameters. One can change or alter the constructed part.

Author Contributions: Methodology, A.M. and M.A.; software, A.M., M.A. and T.N.; formal analysis, A.M., M.A., K.T.M., M.K. and T.N.; writing, original draft preparation, A.M., M.A. and M.K.; Investigation, A.M., M.A. and T.N.; writing, review and editing, A.M., M.A., K.T.M., M.K. and T.N.; visualization, A.M., M.A., K.T.M., M.K. and T.N.; resources, M.A. and K.T.M.; supervision, A.M., M.A. and K.T.M.; funding acquisition, M.A., and K.T.M. All authors have read and agreed to the published version of the manuscript.

Funding: This work was supported by JST CREST Grant Number JPMJCR1911. It was also supported by JSPS Grant-in-Aid for Scientific Research (B) Grant Number 19H02048 and JSPS Grant-in-Aid for Challenging Exploratory Research Grant Number 26630038.

Acknowledgments: The authors would like to thank the anonymous referees for their careful reading of this manuscript and also for their constructive suggestions which considerably improved the article.

Conflicts of Interest: The authors declare no conflict of interest.

\section{Abbreviations}

The following abbreviations are used in this manuscript:

2D two dimensional

3D three dimensional

CAD computer-aided design

CAM computer-aided manufacturing

RDF Radial bases functions

NURBS non-uniform rational B-spline 
DICOM Digital Imaging and Communications in Medicine

ICP iterative closest point

$C^{2} \quad$ parametric continuity of degree one

\section{References}

1. Eufinger, H.; Wehmöller, M.; Machtens, E.; Heuser, L.; Harders, A.; Kruse, D. Reconstruction of craniofacial bone defects with individual alloplastic implants based on CAD/CAM-manipulated ct-data. J. Cranio-Maxillofac. Surg. 1995, 23, 175181. [CrossRef]

2. Müller, A.; Krishnan, K.G.; Uhl, E.; Mast, G. The application of rapid prototyping techniques in cranial reconstruction and preoperative planning in neurosurgery. J. Craniofacial Surg. 2003, 14, 899-914. [CrossRef] [PubMed]

3. Sauret, V.; Linney, A.; Richards, R. Computer assisted surgery: The use of digital images in enabling computerized design and manufacture of titanium implants. Imaging 2002, 14, 464471 [CrossRef]

4. Wu, T.; Engelhardt, M.; Fieten, L.; Popovic, A.; Radermacher, K. Anatomically constrained deformation for design of cranial implant methodology and validation. In Medical Image Computing and Computer-Assisted Intervention MICCAI; Springer: Berlin/Heidelberg, Germany, 2006; p. 916.

5. Shui, W.; Zhou, M.; Deng, Q.; Wu, Z.; Duan, F. 3D craniofacial reconstruction using reference skull-face database. In Proceedings of the 25th International Conference of Image and Vision Computing New Zealand (IVCNZ), Queenstown, New Zealand, 8-9 November 2010.

6. Carr, J.C.; Fright, W.R.; Beatson, R.K. Surface interpolation with radial basis functions for medical imaging. IEEE Trans. Med Imaging 1997, 16, 96-107. [CrossRef] [PubMed]

7. Carr, J.C.; Beatson, R.K.; Cherrie, J.B.; Mitchell, T.J.; Fright, W.R.; McCallum, B.C.; Evans, T.R. Reconstruction and representation of 3D objects with radial basis functions. In Proceedings of the 28th Annual Conference on Computer Graphics and Interactive Techniques, Los Angeles, CA, USA, 12-17 August 2001; ACM: New York, NY, USA, 2001; pp. 67-76.

8. Majeed, A.; Piah, A.R.M.; Rafique, M.; Abdullah, J.Y.; Rajion, Z.A. NURBS curves with the application of multiple bones fracture reconstruction. Appl. Math. Comput. 2017, 315, 70-84. [CrossRef]

9. Majeed, A.; Piah, A.R.M.; Yahya, Z.R.; Abdullah, J.Y.; Rafique, M. Construction of occipital bone fracture using B-spline curves. Comput. Appl. Math. 2018, 37, 2877-2896. [CrossRef]

10. Majeed, A.; Piah, A.R.M. Image reconstruction using rational Ball interpolant and genetic algorithm. Appl. Math. Sci. 2014, 8, 3683-3692. [CrossRef]

11. Chowdhury, A.S.; Bharkar, S.M.; Robinson, R.W.; Yu, J.C. Virtual Craniofacial Reconstruction using Computer Vision, Graph Theory and Geometric Constraints. Pattern Recognit. Lett. 2009, 30, 931-938. [CrossRef]

12. King, R.E.; Scianna, J.M.; Petruzzelli, G.J. Mandible fracture patterns: A suburban trauma center experience. Am. J. Otolaryngol. 2004, 25, 301-307. [CrossRef] [PubMed]

13. Pascoletti, G.; Calì, M.; Bignardi, C.; Conti, P.; Zanetti, E.M. Mandible Morphing Through Principal Components Analysis. In International Conference on Design, Simulation, Manufacturing: The Innovation Exchange; Springer: Cham, Switzerland, 2019; pp. 15-23.

14. Dirk, V.; Peter, C.; Dirk, L.; Sven, D.G.; Guy, W.; Paul, S. Computerized craniofacial reconstruction using CT-derived implicit surface representations. Forensic. Sci. Int. 2006, 159, S164-S174.

15. Klein, R.; Schilling, A.G.; Strasser, W. Reconstruction and simplification of surfaces from contours. Graph. Model. 2000, 62, 429-443. [CrossRef]

16. Zheng, G.; Rajamani, K.T.; Nolte, L.P. Use of a dense surface point distribution model in a three-stage anatomical shape reconstruction from sparse information for computer-assisted orthopaedic surgery: A preliminary study. In ACCV06, Lecture Notes in Computer Science; Springer: Berlin/Heidelberg, Germany, 2006; Volume 38, pp. 52-60.

17. Amba, D.B.; Ravi, M.W. Reverse Engineering of Human Body: A B-spline based Heterogeneous Modeling Approach. Comput.-Aided Des. Appl. 2008, 5, 194-208.

18. Kou, X.Y.; Tan, S.T. Heterogeneous object modeling: A review. Comput. Aided Des. 2007, 39, $284-301$. [CrossRef]

19. Lian, Q.; Li, D.C.; Tang, Y.P.; Zhang, Y.R. Computer modeling approach for a novel internal architecture of artificial bone. Comput.-Aided Des. 2006, 38, 507-514. [CrossRef] 
20. Ball, A.A. CONSURF I: Introduction of the conic lofting tile. Comp. Aided Des. 1974, 6, 243-249. [CrossRef]

21. Goodman, T.N.; Said, H. Properties of generalized Ball curves and surfaces. Comput.-Aided Des. 1991, 23, 554560. [CrossRef]

22. Farin, G.E. Curves and Surfaces for CAGD: A Practical Guide; Morgan Kaufmann: Burlington, MA, USA, 2002.

23. Salomon, D. Curves and Surfaces for Computer Graphics; Springer Science and Business Media: Berlin/Heidelberg, Germany, 2007. 